\title{
On the Functional Approach to Absolute Constructions in Scientific Prose Style (With Special Reference to Engineering Research Articles)
}

\begin{abstract}
Dr. Minoo Khamesian
NIT University, Babol, Iran

To be able to learn and use English, the lingua franca of science and technology, for effective international communication, becoming acquainted with the basic language of one's profession seems to be crucially important. In this respect, written academic discourse is a considerably broad notion requiring consideration of various aspects both on the linguistic and extra-linguistic planes. The present work linguostylistically (on both semantic and metasemiotic levels) analyzed the functional aspect of using absolute constructions, a predicative construction in which non-finite forms of the verbs stand in predicate relation to their overt subject, in technical writing. The results revealed that the distinctive morphosyntactic structure of absolute constructions, although purely linguistic, would provide to serve functions far beyond linguistics proper. Otherwise stated, absolute constructions being concise and laconic are capable of communicating complete informative line within a sentence, giving an opportunity to fit more information into a smaller volume. Interestingly, contrary to widely believed notion, the preposition of them rather than postposition, preferred in the scientific register, could be allocated to their rhetorical impact. In addition, due to their frequency in this style, they need to be paid their deserved attention while teaching EAP to engineering students.
\end{abstract}

Keywords: absolute constructions, functional style, engineering research articles

\section{Introduction}

Written academic discourse tends to be a very broad notion requiring consideration of various aspects both on the linguistic and extra-linguistic planes. On the other hand, the grammatical structure of any sentence includes the peculiarity of being concealed in an authentic unit of communication which constitutes a complicated integrity of speech, language, and thought, hence the correlation of semantic and formal aspects of a sentence. Communities of practice are considered intricate collective networks sharing common knowledge, linguistic register, and social values and conventions. Engineering research articles are a particular example of a specialist register used by a very restricted community of users (Trimble, 1985; Swales, 1990; Bhatia, 1993).

The correlation between form and function has been outlined by the functionalist approach to the description and explanation of language, ${ }^{1}$ according to which all linguistic units serve to fulfill certain functions apart from structural peculiarities.

Dr. Minoo Khamesian, linguist, EAP lecturer, and freelance editor, Ph.D. in Linguistics, Basic Sciences, NIT University.

${ }^{1}$ See for example Vinogradov, V. V. (1963). Stylistics, theory of the poetic speech, poetics. Moscow: APN USSR. 
The language of science is concerned responsible for determining the principles governing the physical universe. Approaching this end depends to a large extent on how the language is used. As a consequence, authors of scientific works who wish to make their own contribution in this regard should satisfy the same linguistic constraints if their work is to be correctly interpreted and accepted by their peers. In other words, academic English, as used in the scientific domain of knowledge, represents an instance of how cognitive, linguistic, and discourse features characterize particular social arrangements, and an example of how language in general is, "a social practice determined by social structures" (Fairclough, 1989, p. 17).

In this study, it was attempted to draw certain conclusions concerning absolute constructions since undoubtedly the syntactic organization of texts tends to be an indicator of interrelation and complementarity of form and function.

As known, in the study of syntax, the phenomenon of semi-composite sentences has established a prominent seat for itself. To further explain, they are sentences which do not completely fit into simple or composite due to their peculiar structures. Having two predicative lines, one is the main clause; however, the other would extend it through incomplete secondary predication, not having a finite verb.

We should hasten to add that syntagm is the linear correlation of sentence elements, whereas through semantics the extralinguistics are realized. On the other hand, modality is the correlation of content and extralinguistic reality of a sentence with respect to grammatical tense and mood on behalf of the communicator of a message, all of which channel us to the category of predication. This is what unites and synthesizes the aforementioned aspects of a sentence, i.e., linking reality to its linguistic realization. It is even said to have the role of making up the meaning of a sentence (Karapetyan, 2009).

\section{Analysis}

Keeping an eye on the structural peculiarities, we should not ignore certain functions linguistic units tend to fulfill. That language is a powerful means of communication of information and experience transmission among discourse communities is undeniable. As Halliday (1985) puts, "Language is as such because of the functions it has evolved to serve" (p. 45).

Following Functional Approach, the syntactic organization of texts will serve an indication of interrelation and complementary of form and function, being familiar with which would obviously promote further communication progress in the given area.

To conduct the analysis, a corpus of approximately 300 pages of engineering research articles of different spheres was used, all of which were successfully published in international journals, available online. Our analysis first dwells on the morphosyntactic peculiarities of Absolute Constructions, i.e., form comes first.

\section{Examples}

- Using a traditional approach, the stability of large earthen dam in static condition is studied with the limit equilibrium method.

- Taking into account $\mathrm{Cu}, \mathrm{Pb}, \mathrm{Zn}, \mathrm{Cd}$, and Fe, the PI and the EF were calculated.

- The generated firing pulses connected to VSI, it can convert the required voltage levels even under faulted conditions too.

- Depending on the frequency range of interest, the hydraulic system, body panels, steering column, and other vehicle components can also become active. 
According to Crystal (1987) who speaks in favour of the formal definition of the verb,

The formal definition of a verb refers to an element which can display Morphological contrasts of Tense, Aspect, Voice, Mood, Person, and Number. Functionally, it is the element, which singly or in combination with other verbs (i.e., "verb phrase"), is used as the minimal Predicate of a sentence, co-occurring with a Subject, e.g., he/came. (p. 325)

However, the underlined sections are considered the secondary predication dependent to the primary, the trait provided by the morphological or formal peculiarities of non-finite verbs.

The correlation of form and content being very intricate and controversial could be in particular manifested in the contradiction of grammatical arrangement of utterances and their semantic structure. The indirect nomination of reality as Karaptyan (2009) puts, "is marked by the breach of the isomorphism between the syntactic organization of the utterance and the essence it expresses" (p. 210). This might result in certain elements or components of the reflected reality not being shaped in words, i.e., implicitly, which would precondition diverse shifts in the grammatical structure and facilitate a wider scope to form variable syntactic units. That is why it would be more expedient to regard Absolute Constructions as the semantic formation of syntax with implicit predication. As we can see, the Absolute Construction can join the independent main clause, yet preserving relative autonomy which can be expressed by means of punctuation in writing, or intonational delimitation in speech and the communicative stress.

The peculiarity of such constructions, i.e., including secondary predication is that their status in both semantic and syntactic hierarchy is lowered. We should hasten to add that their capability of expressing a complete idea, being peculiar of sentences, without completing the syntactic status of a sentence would make it possible to concentrate a number of predicative lines, hence several assertions, within the framework of the same sentence.

Take, for example:

- The first step is to establish the in situ stress state conditions using SIGMA/W that exist before the earthquake occurs, using linear elastic model and nonlinear (elastic-plastic) model respectively.

This is a beneficial feature with an exclusive role in speech development, which should not be underestimated in teaching EAP as they constitute a considerable percentage in scientific writing in general and in technical writing as well.

As a consequence, the two-fold formal characteristic of Absolute Constructions is worth being paid attention, i.e., being really concise and laconic (the property provided by the morphological, in other words, formal peculiarities of non-finite verbs/word groups as Sweet 1903 puts it), these constructions are able to communicate complete informative line within sentences, as well as the logical connection between the linguistic units and the nuclear or the main clause they modify.

As far as the former is concerned, it does not seem unreasonable to say that quantitative research so far has shown abundant use of these structures in scientific register in general. Engineering research articles analyzed here showed not to be an exception since on average we encountered one in every six sentences. This could rely upon extralinguistic factors, i.e., the objectives being sought by science. To further explain, the ever-increasing rhythm of advances in science and technology would undoubtedly call for information transmission in the framework of linguistics as concise and compressed as possible. This is in contrast with fiction and literary texts proved more sophisticated, and seasoned, within which subtle linguistic means (tropes and figures of speech) for the expression of reality would be favoured over laconism and conciseness. 
As a consequence, these constructions are fully capable of accomplishing the object of transmitting data in the shortest possible time due to their structural brevity, a feature which, nonetheless, would not distort what they intend to communicate. As known, the interrelation of form and function is inseparable and constitutes a duality which needless to say would require a thorough consideration of both for comprehensibility and felicitous explanation of grammatical phenomena, Absolute Constructions here.

This can bring the assumption that ACs (for short) as distinct representatives of construction with secondary predication would vary from specification and concession to time and place indication, i.e., adverbials.

Below are some corresponding examples adapted from the articles investigated to substantiate the assertion:

- Using the superposition principle of force, figure...can be decomposed into figure... and figure...cases.

- Considering these values and taking into account that gross values of $\mathrm{Cu}, \mathrm{Zn}, \mathrm{Cd}$, and $\mathrm{Pb}$ were higher than the concentrations in the earth's crust, one is able to say that the waters of these lakes were enriched with heavy metals.

- Depending on the execution location, power control algorithms can be categorized as either centralized or distributed.

- The voltage source converter having six IGBT's, these are performed to convert dc voltages into ac voltages.

- With the standard base, the estimation of the yield is changed by estimation of the blunder hysteresis controller rupture and determination of the tenet base is done utilizing experimentation systems and is likewise done through validations.

- With any kind of knowledge representation scheme, it is possible to infer knowledge that is not directly represented by the scheme.

As we can see in the examples, and the examination of the corpus showed as well, the adverbials in this register would generally establish cause-effect relationship as well as specifying and explaining instrumentation or condition, a feature in agreement with Kortmann (1990) (Table 1 in Appendix shows the result of his investigation). However, regarding the Functional Approach we can explain that as the ultimate goal of informative communication is persuasion, i.e., presenting one's point-of-view and/or suggesting a commentary, these functions of adverbials would not distort the function of the message which calls for "clarity of purpose" and "lucidity of exposition".

Another criterion worth considering is the position of Absolute Constructions in the sentence. According to Chubaryan and Karapetyan (2007), the preferred position of these constructions in the scientific register would be that of postposition, where the explanation of facts can be sought in the peculiarities of the functioning they are summoned to fulfill.

The clarity and explicitness of a statement is considered the foremost condition in scientific communication. This takes us to the theory of functional sentence perspective with regard to the theme, i.e., given information, and the rheme, i.e., new information of a message to be conveyed. ${ }^{2}$ Otherwise stated, it is presupposed in scientific writing to encounter a predominant number of ACs in postposition, where they can be clearly interpreted; as known, the clarity and explicitness of a statement is the foremost condition in the

2 See Halliday, M. A. K. (1985). An introduction to functional grammar. Longman. 
so-called felicitous communication in scientific discourse. Surprisingly, contrary to this common belief, in our analysis preposition was predominant. This would breach the feature of clarity in this style, i.e., readability formula. Is it born out of necessity? Of course not. Technical writing is known to be hard to read due to abundant use of technical words. As a consequence, we should say this characteristic would make it even more difficult to decipher, especially to non-native learners.

Therefore, it does not seem unreasonable to say that in the corpus investigated, i.e., articles since 2012, the postposition of absolute constructions would not manifest its probable linguistic structure, thus not performing the function they are summoned to realize, readability (to reiterate).

\section{Conclusion}

How to use English in science and technology to communicate plainly and readably would undoubtedly require certain compositional strategies, from the level of words and phrases to that of sentences and even paragraphs.

Our analysis on the form and function of ACs revealed that the abundant use of them in engineering research articles would fulfill some objectives, i.e., linguistic brevity, which would undoubtedly give the chance to the author to meet the ever-increasing rhythm of advances in science and technology which calls for information transmission in the framework of linguistics as concise and compressed as possible. Nevertheless, they could be opaque to EAP learners of some non-cognate languages, e.g., Iranian learners. With respect to the predominance of preposition of ACs in the analyzed style, which can be considered a novel peculiarity replacing the theme/rheme syntagm of a sentence, we could pinpoint the globalization of scientific knowledge which has led to the clash of various cultures and languages in the academic environment displayed mainly on the linguistic plane. English serving as the lingua franca of science and technology is considered the medium for disseminating scientific findings throughout the globe, exchanging opinions, as well as supporting or rejecting hypotheses. Hence, not bizarre to say that to a certain extent "domain specific English", as Gasparyan (2011, p. 19) puts it, could absorb cultural and linguistic peculiarities of scholars of different nationalities.

\section{References}

Bhatia, V. J. (1993). Analysing genre: Language use in professional settings. Harlom: Longman.

Chubaryan, A., \& Karapetyan, R. (2007). The English absolute constructions in the functionalist theory perspective. Foreign Languages in Higher Education 8, Yerevan: YSU Publishers.

Crystal, D. (1987). The English language: A guided tour of the language. Harmondsworth: Penguin.

Fairclough, N. (1989). Language and power. London: Longman.

Gasparyan, S. (2011). The Linguostylistic and linguopoetic analyses in action. Gitelick, 16, 38-44.

Halliday, M. K. A. (1985). Introduction to functional grammar. London: Longman.

Karapetyan, R. (2009). On the syntactic status of absolute constructions. Armenian Folia Anglistika, International Journal of English Studies, 1-2(6), 208-214.

Kortmann, B. (1985). Adverbial participle clauses in English. London: Routledge.

Swales, J. M. (1990). Genre analysis: English in academic and research settings. Cambridge: Cambridge University Press.

Sweet, H. (1903). New English grammar, logical and historical. Oxford: Oxford University Press.

Trimble, L. (1985). English for science and technology. Cambridge: CUP. 
Appendix

Table 1

\begin{tabular}{lll}
\hline Most informative & Least informative \\
\hline Concession & & Manner \\
Contrast & & Exemplification \\
Condition & Purpose & Specification \\
Instrument & Result & Simultaneity \\
Cause & Posteriority & Accompanying circumstances \\
Anteriority & & \\
\hline
\end{tabular}

